\title{
Near-infrared adaptive optics dissection of the core of NGC 1068 with NAOS-CONICA ${ }^{\star}$
}

\author{
D. Gratadour ${ }^{1,2}$, D. Rouan ${ }^{1}$, L. M. Mugnier ${ }^{2}$, T. Fusco ${ }^{2}$, Y. Clénet $^{1}$, E. Gendron ${ }^{1}$, and F. Lacombe ${ }^{1}$ \\ 1 LESIA, Observatoire de Paris, 5 place Jules Janssen, 92195 Meudon, France \\ e-mail: dgratadour@gemini . edu \\ 2 DOTA-ONERA, Av. de la division Leclerc, Châtillon, France
}

Received 15 October 2004 / Accepted 1 September 2005

ABSTRACT

We present an update analysis of recent near-infrared adaptive optics observations of NGC 1068 obtained with NAOS-CONICA at VLT/UT4. $K \mathrm{~s}, L^{\prime}$ and $M^{\prime}$ bands images were deconvolved using MISTRAL, a regularized algorithm based on a maximum a posteriori estimation of the object. Two regularization methods, one including a new maximum likelihood estimation of the object Power Spectral Density, and an edge preserving one, have been tested and converge to consistent results. The deconvolved images show a coherent evolution of the IR emission from 2.2 to $4.8 \mu \mathrm{m}$. Deconvolution brings new elements: a) it strengthens the very peculiar nature of the four parallel elongated nodules previously discovered along the jet, which appear unresolved perpendicular to their long axis; b) it underlines the strong correlation between UV clouds and IR features, and c) it provides a more accurate multi-wavelength registration of the actual active nucleus. The overall aspect of the central $1^{\prime \prime} \times 1^{\prime \prime}$ IR emission seems to point to the jet as a major mechanism to shape the NLR. For each identified structure, we derive a color temperature now based on three bands $(M, L$ and $K)$, before and after deconvolution, confirming the need for clumps of dust at unexpectedly high and almost constant temperature (about $500 \mathrm{~K}$ ) up to 70 pc north of the nucleus. We explore several mechanisms to explain the color temperature and show that shocks, induced for instance by the interaction of the jet with a giant cloud, is unlikely to be the dominating mechanism to heat the dust. We detail our model of transient heating of Very Small Grains and show that it can provide a consistent explanation of the $K, L, M$ colors and their lack of variation with distance when $0.6 \mathrm{~nm}$ diamond-like grains are heated by 4 to $8 \mathrm{eV} \mathrm{UV}$ photons. However, we do not exclude the possibility that part of the excitation could come from shocks. At $K \mathrm{~s}$, deconvolution reinforces the previous claim that the central core is partially resolved along the N-S direction: the best fit to our data is an elliptical Gaussian extended along PA $=-16$ with a $2.1 \mathrm{pc} F W H M$ along this direction. This result agrees with the predictions of the radiative transfer model we previously developed to interpret the spectroscopic behavior at $K$, and is consistent with VLTI/VINCI measurements. Several questions are raised by this study: a) is the jet dominant in shaping the NLR of this AGN? b) what is the real state of the dust in the environment of the core; c) is the simple doughnut torus model able to explain IR emission of the central source with a morphology that appears increasingly complex at small scale?

Key words. galaxies: Seyfert - galaxies: individual: NGC 1068 - infrared: galaxies - techniques: image processing - radiative transfer galaxies: jets

\section{Introduction}

The mechanisms shaping and powering the cores of Active Galactic Nuclei (AGN) are not yet entirely characterized. Their distances to us make any of our high angular resolution observations inadequate. As one of the closest active nuclei $(15 \mathrm{Mpc}$, $\left.1^{\prime \prime}=70 \mathrm{pc}\right)$, NGC 1068 is considered as one of our best test subjects, and its intensive study during the past two decades, over all bands of the electromagnetic spectrum, as helped to make steps forward. The standard model of AGN assumes a very massive black hole (Krolik 1999; Elvis 2000) with an accretion disk and possibly a jet as the source of the powerful continuum. A way to unify the entire taxonomy of activity types involves anisotropic distribution of optically thick

^ Based on observations collected at the ESO/Paranal YEPUN telescope, Proposal 70.B-0307(A). material around the central engine. The viewing angle would modify the aspect of the same kind of object, exhibiting broad lines when viewed face on, and only narrow lines when the core is obscured. The question of the size and morphology of this obscuring structure is still a matter of debate (Nenkova et al. 2002; Fadda et al. 1998), and since the discovery of broad polarized lines in NGC 1068 (Antonucci \& Miller 1985) many attempts have been made to test Seyfert unification.

NGC 1068 is the archetypal Seyfert II nucleus, meaning that obscuring material hides the Seyfert I activity of the core. It seems to possess all the characteristics of a classical AGN. First, the powerful UV-Xray central source is supposed to heat its environment, hidden in the UV-visible, but recent X-ray observations with HETGS (Ogle et al. 2003) detected Fe emission consistent with re-processing of nuclear light, and favored photoionization as the main source of excitation. Moreover, 
polarization maps at UV (Capetti et al. 1995) show a very localized polarization center at the lower edge of one of the identified NLR clouds (cloud B). A radio jet (Gallimore et al. 1996 b) is probably interacting with a giant molecular cloud at $0.6^{\prime \prime}$ north of the nucleus (Gallimore et al. 1996a). Radio observations also identify maser emission (Gallimore et al. 2001), probably tracing the inner edges of the warm torus, pumped by the UV continuum of the central source, and whose periodicity is consistent with a rotating disk geometry. Jet masers are also detected, probably excited by shock-generated $\mathrm{X}$ rays where the jet interacts with the cloud. A starburst ring has been observed at a distance of $1 \mathrm{Kpc}$ from the nucleus (Davies et al. 1998) and a wrapped molecular disk is suspected in the millimeter range in the inner 300 pc (Schinnerer et al. 2000), as well as at near-IR (Alloin et al. 2001). Modeling of the kinematics suggests a bar driven motion and an enclosed mass of $10^{8} M_{\odot}$ in the inner $25 \mathrm{pc}$, which provides another clue to the presence of a very massive black hole in the core. A ionization cone is detected from optical to UV (Evans et al. 1991; Macchetto et al. 1994), in the direction of the jet. It consists of a patchy distribution of high velocity clouds and filaments probably ablated from molecular clouds by the nuclear emission. Intensive HST spectroscopic studies in the UV (Crenshaw \& Kraemer 2000a,b; Kraemer \& Crenshaw 2000a,b; Cecil et al. 2002) have placed strong constraints on physical conditions in the NLR. A N-S elongated structure is detected in the mid-IR (Bock et al. 2000) as well as in the thermal IR adaptive optics images of Marco \& Alloin (2000). Deconvolved diffraction limited near-IR images of Rouan et al. (1998) also show a N-S elongated structure and possible traces of the putative torus and a micro-spiral structure. More recently, adaptive optics $K$-band spectroscopy (Gratadour et al. 2003) and comparison with a numerical model allowed us to place some constraints on the nature and orientation of the obscuring material.

Speckle observations in the $H$ and $K^{\prime}$ band (Weigelt et al. 2004) show an elongated central source along PA $=16^{\circ}$ with a size of $1.3 \times 2.8 \mathrm{pc}$ at $K^{\prime}$. Recent interferometric observations have placed constraints on the size of the central source in the IR. Jaffe et al. (2004) fitted their $10 \mu \mathrm{m}$ VLTI/MIDI observations with a double Gaussian component: a hot component $(T=800 \mathrm{~K}$ ) with a $0.7 \mathrm{pc} F W H M$ along the direction of the jet, and a warm one $(T=320 \mathrm{~K})$ with a size of $2.1 \times 3.4 \mathrm{pc}$, elongated in the direction perpendicular to the jet axis. As they used two different baselines, their measurements were only made along PA $=2^{\circ}$ and PA $=45^{\circ}$. VLTI/VINCI observations of Wittkowski et al. (2004), with one baseline along PA $=45^{\circ}$ were also fitted with a double Gaussian component with part of the flux coming from a compact source smaller than 5 mas (0.4 pc).

The images used for the present work are based on observation previously reported in Rouan et al. (2004) and Gratadour et al. (2005b), hereafter RLG04 and GRB04. In Sect. 2 we describe data acquisition and data reduction procedures developed to recover the theoretical diffraction limited resolution of these IR A.O. observations. Then the deconvolution process, in which a new regularization criterion has been tested, is described and the results are discussed. In Sect. 3, a photometric and morphological study of deconvolved images is developed, in which we propose a multi-wavelength registration from UV to radio and an interpretation of the features observed. In Sect. 4, we compare these observations to different mechanisms responsible for dust heating in the NLR and we summarize our conclusions in the last section.

\section{Observations and data processing}

\subsection{Data acquisition}

The observations were performed using $\mathrm{NaCo}$ at the Nasmyth focus of YEPUN (Rousset et al. 2000; Lenzen et al. 1998) during the nights 18-26 November 2003. Two sequences of 20 images of a reference star and one sequence (in between) of 40 images of the galaxy were acquired in each band $(K \mathrm{~s}$, $L^{\prime}$ and $M^{\prime}$ ). The Auto-jitter mode was used so that each frame is randomly translated in the plane of the sky within a box of $8^{\prime \prime} \times 8^{\prime \prime}$. A reference source, of the same equivalent magnitude as the nucleus of NGC 1068, was chosen to ensure the same performance of the A.O. system. During the observations of NGC 1068, the Strehl ratio obtained ranged from 0.5 to 0.7 , and during the reference acquisition, up to 0.6. The airmass ranged from 1 to 1.2 and the seeing was fairly good (about $0.8^{\prime \prime} \pm 0.2$ during the observations of NGC 1068). Since the nucleus of this galaxy is bright enough, the visible wavefront sensor of $\mathrm{NaCo}$ was used in the most accurate mode $(14 \times 14$ sub pupils $)$. The very high correction level leads to diffraction limit resolution in all bands as shown on images of RLG04. The Ks band image was acquired with pixels of 13 mas and the $L^{\prime}$ and $M$ band images with pixels of 27 mas.

\subsection{Data reduction}

Data reduction was carefully done using improved softwares dedicated to A.O. image processing. After a classical flat-field correction, the bad pixel correction is important since an exhaustive elimination is required for the next stages of the process. Bad pixels are hot, dead or "mad" (i.e. not responding each time). Most of them appear on every image and so can be detected on an image acquired under homogeneous illumination (flat-field image for instance). They are then corrected by applying a median filter in a box of a tunable size around each bad pixel. Big groups of dead pixels can also be corrected using a refined median filter. After this rough correction, we need to eliminate residual mad pixels and cosmics. They can be characterized as producing high spatial frequencies that cannot be transmitted by the telescope. Since the images are at least Nyquist sampled, all the information coming from the telescope can be suppressed in the image by applying a high-pass frequency mask at $\frac{D}{\lambda}$ (with $\lambda$ the central frequency of detection and $D$ the telescope diameter) in the Fourier space. The local maxima of the cross-correlation function of the residual image and the Fourier transform of the mask give the positions of the bad pixels. The detection can be thresholded to a tunable number of sigmas. The selected pixels are then corrected by applying a median filter as described above. This new detection method can be used as an automated process to remove 
cosmic rays and residual mad pixels, after a first correction using a map of dead pixels deduced from the flat-field image.

Sky background is then subtracted from each image by selecting another image of the jittered sequence, close enough in time to ensure good sky subtraction, but far enough in the plane of the camera to minimize the NGC 1068 galactic background.

Finally, the sequence of images has to be recentered very accurately to keep the spatial resolution when averaging the frames. To estimate the translation parameters between the images, a new maximum likelihood algorithm was used that allows one to recenter images at the level of the tenth of pixels, even with very noisy images, as in the case of thermal infrared imaging. The criterion

$\mathcal{J}\left(x_{k}, y_{k}\right)=\sum_{x, y} \frac{1}{2 \sigma_{k}^{2}(x, y)}\left|i_{k}(x, y)-i_{0}\left(x-x_{k}, y-y_{k}\right)\right|^{2}$

is minimized for each image $i_{k}(k=1 \ldots N)$ of the sequence of $N$ images, with $i_{0}$ the image chosen as a reference and $\sigma_{k}^{2}$ the noise variance in $i_{k}$, which can be estimated on the image (Mugnier et al. 2004). This registration method, which notably outperforms the classical cross-correlation, is fully described in Gratadour et al. (2005a), as is its performance in various noise regimes. It has also been tested on $\mathrm{L}^{\prime}$ band $\mathrm{NaCo}$ images of $\mathrm{Sgr}$ $A^{*}$ (Clénet et al. 2005), showing the same kind of efficiency on images that have about the same SNR.

Final images, obtained after averaging the recentered frames, are displayed in Fig. 1. of RLG04 for the $L^{\prime}$ and $M^{\prime}$ band and Fig. 2 of GRB04 for Ks. Some images of each sequence have not been selected when averaging because of poor $\mathrm{S} / \mathrm{N}$ or not optimal atmospheric correction. The airy rings appearing on $L^{\prime}$ and $M^{\prime}$ band images of NGC 1068 and the reference star demonstrate that diffraction limited resolution was actually achieved.

\subsection{Deconvolution of reduced data}

We performed deconvolution using as the PSF the image of the reference source, which has been carefully chosen to reproduce the same A.O. performances as in the case of the galaxy.

We selected the frames in both sequences (object and reference star) that where acquired with a similar Strehl ratio as evaluated by NAOS (Fusco et al. 2004), which leads us to keep more than $60 \%$ of the frames in both cases, if we consider a range of 5\% in Strehl.

\subsubsection{Methods}

Images were deconvolved using the image restoration algorithm MISTRAL (Conan et al. 1998; Mugnier et al. 2004). Two variants have been used and compared in order to test the reliability of the results.

This deconvolution method consists of minimizing a two term expression: one measures the fidelity to the data and the other one describes a prior knowledge of the object (the regularization term). The latter represents any prior knowledge of the object. Its expression thus depends on the shape of the object (strong, smoothed edges) and peculiarities (extended, point-like, disc-like, etc.). Several criteria can be used and we compared two: a classical linear-quadratic (L1-L2) regularization (Mugnier et al. 2004), and a quadratic one, which needs an estimate of the object PSD. The latter can be estimated directly on the final image, using the PSF, as described in Appendix A.

\subsubsection{Results}

The actual resolution in the images after deconvolution is not predictable. However, as the central source is clearly unresolved at $\mathrm{M}$ band before deconvolution, the FWHM of this source after deconvolution would give an estimate of the final resolution. It is found to be 1.7 pixels, or $2.8 \mathrm{pc}$ at the distance of NGC 1068 , in the $L^{\prime}$ and $M$ band images and $1.3 \mathrm{pc}$ in the $K$ s image.

As the regularization term is object dependent, the same deconvolution method cannot be applied to the PSF in order to check for hypothetical fake structures.

The results obtained with the two methods are very similar, as shown in Figs. 1-3 in each band. The PSD-type regularization term was used first to give a preliminary estimate of the final shape of the object, as the only parameter to tune is the weight of the regularization. Then the two parameters of the L1-L2 method are adjusted to reproduce the same overall shape while sharpening the nuclear region. This leads to very different parameters in each bands because of the differences in the initial images. For $K \mathrm{~s}$, the hyper-parameter value must be high to avoid any ringing effect, when deconvolving with the quadratic regularization term. We think it is due to an imperfect knowledge of the PSF. Deconvolved images in different bands can be superimposed (Figs. $4 \mathrm{~b}$ and c) with an excellent correspondence of the sharp structures, giving good confidence in the final result in terms of morphology. Moreover, many of the structures found in deconvolved $K$ s images can be identified with structures detected in coronagraphic observations (GRB04): spot N1, the shape of structure IR-1b and also the structure IR-6.

After frame selection, as the Strehl ratio obtained with the object and with the star are very close $( \pm 5 \%)$, the reference star image is a very good calibration of the instrument PSF during the observations and myopic deconvolution is apparently not needed in this case, except for $K$ s.

\section{General results}

\subsection{Photometry}

We performed photometry on data before deconvolution. Table 1 gives the color index found in all identified regions. The measured magnitudes have been calibrated with the star (HD 16835, a F0 star) used as a photometric and PSF reference. It is a 2MASS source, so its $K$ band magnitude $\left(M_{K}=6.705\right)$ is known within $1 \%$. To evaluate the $K \mathrm{~s}-L^{\prime}$ and $K \mathrm{~s}-M$ colors, we have to know the spectral type of this star. As the spectral class is not given in the CDS data base, we have to evaluate it. Based on Hipparcos photometry as well as 2MASS measurements, the color indices are consistent with a main sequence FO star. We therefor took typical colors of a FO V star to 


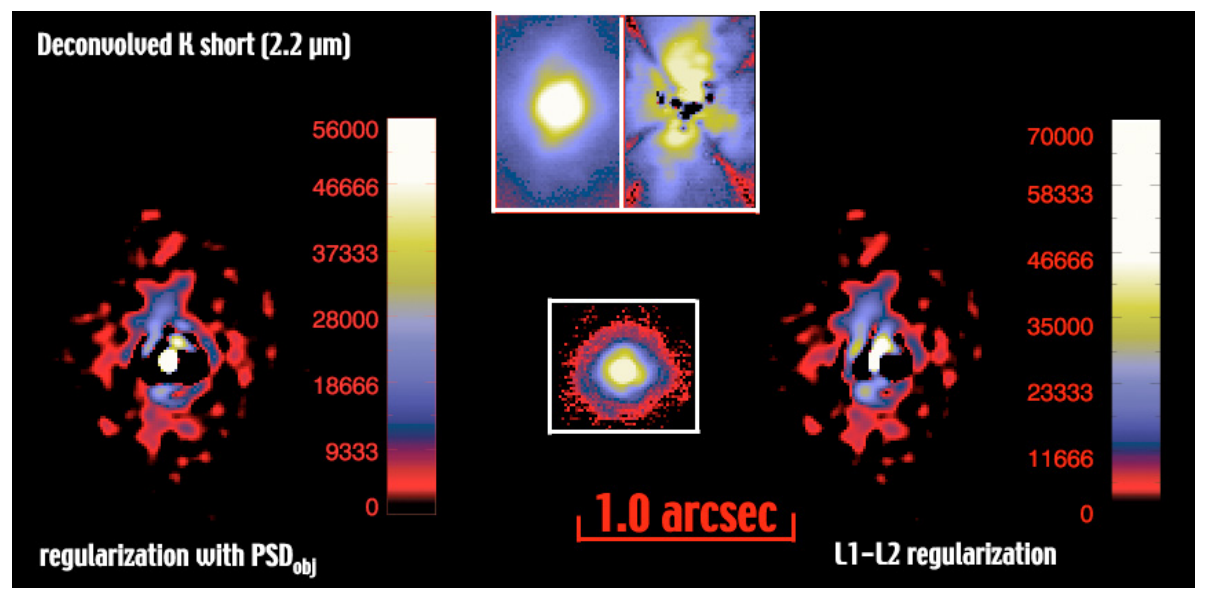

Fig. 1. $K$ short deconvolved images of NGC 1068. Left is the deconvolution with the $\mathrm{PSD}_{\text {obj }}$ regularization term, the weight is set to 500. Right deconvolution with a classical L1-L2 term, the threshold $\delta$ is set to 1 and the hyper-parameter $\mu$ to 5 . The left upper inset is the non deconvolved central source at $K \mathrm{~s}$ and right is the coronagraphic image both from GRB04. The PSF used for deconvolution is shown in the middle inset.

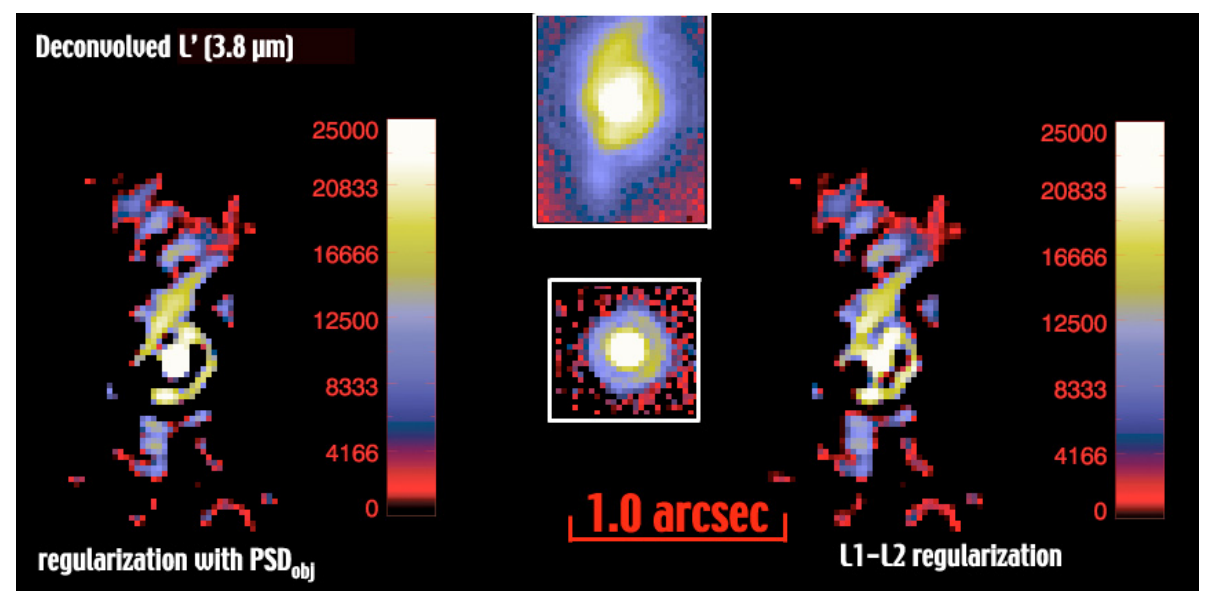

Fig. 2. $L^{\prime}$ deconvolved images of NGC 1068. Left is the deconvolution with the $\mathrm{PSD}_{\mathrm{obj}}$ regularization term, the weight is set to 1 . Right deconvolution with a classical L1-L2 term, $\mu$ is set to 5 while $\delta$ is set to 1 . The upper inset is the un-deconvolved central source from RLG04 and the PSF used for deconvolution is shown in the middle inset.

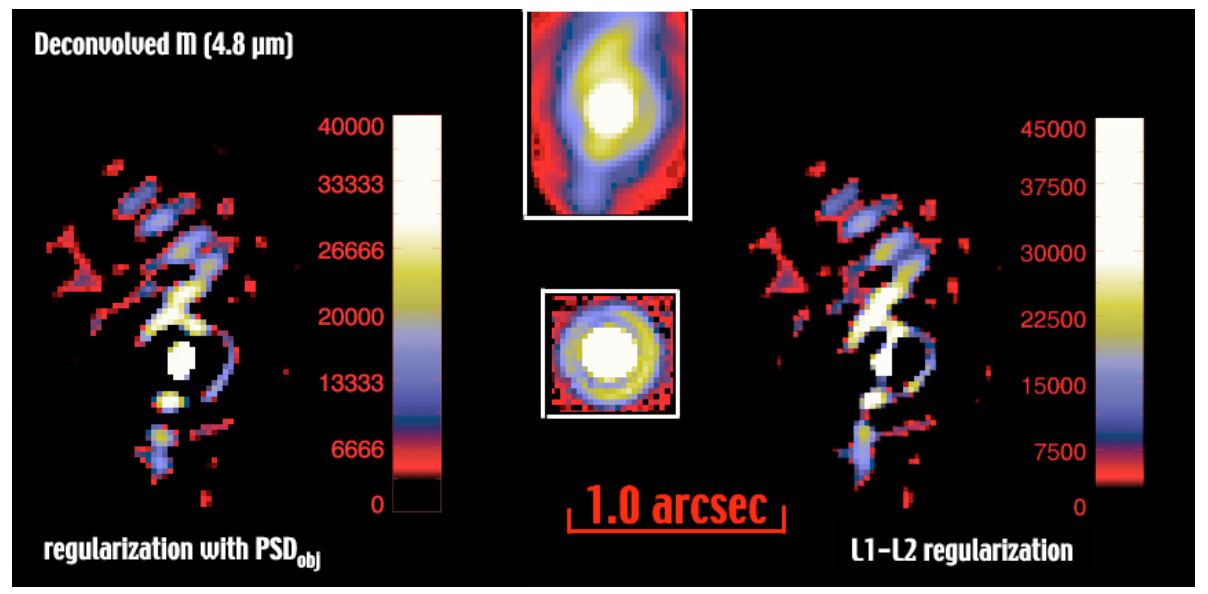

Fig. 3. $M^{\prime}$ deconvolved images of NGC 1068. Same as Fig. 2. With the $\mathrm{PSD}_{\text {obj }}$ regularization term, the weight is set to 1 and with a classical L1-L2 term, $\mu$ is set to 1 while $\delta$ is set to 0.5 .

calibrate the measurements of NGC 1068. If we assume a $1 \%$ error in the color ratio of a main sequence $\mathrm{F} 0$, as well as a $5 \%$ error due to a count estimation error, and considering that a) the star is not variable (as confirmed by Hippparcos data); b) the night was clear; c) similar atmospheric conditions between the reference and the object observations, we 
Table 1. Values of the color index and $K$ s magnitude in each identified region before deconvolution. The $K$ s magnitude of knots have been estimated on coronagraphic images of GRB04. The aperture radius is given in" as well as in pc.

\begin{tabular}{cccccc}
\hline \hline Position & $\mathrm{Ap}\left(^{\prime \prime}\right)$ & $\mathrm{Ap}(\mathrm{pc})$ & $L^{\prime}-M^{\prime}$ & $K \mathrm{~s}-L^{\prime}$ & $K \mathrm{~s}$ \\
\hline nucleus & 0.08 & 6 & 2.2 & 1.1 & 9.9 \\
& 0.13 & 9 & 2.1 & 1.6 & 9.3 \\
& 0.27 & 20 & 1.7 & 2.3 & 8.9 \\
Struct. IR-1b & 0.08 & 6 & 1.2 & 4.1 & 12.0 \\
& 0.13 & 9 & 1.1 & 3.7 & 10.6 \\
IR-6 & 0.08 & 6 & 0.9 & 4.3 & 13.6 \\
& 0.13 & 9 & 1.1 & 4.3 & 12.8 \\
Knot IR-1 & 0.08 & 6 & 1.5 & 4.0 & 14.0 \\
Knot IR-2 & 0.08 & 6 & 1.8 & 4.2 & 14.2 \\
Knot IR-3 & 0.08 & 6 & 1.8 & 4.6 & 14.6 \\
Knot IR-4 & 0.08 & 6 & 2.0 & 4.5 & 15.0 \\
\hline
\end{tabular}

Table 2. Values of the color indices in each identified region after deconvolution. The calibration was made assuming the same color ratio for the central source as found before deconvolution. The aperture radius is given in " as well as in pc.

\begin{tabular}{ccccc}
\hline \hline Position & $\mathrm{Ap}\left(^{\prime \prime}\right)$ & $\mathrm{Ap}(\mathrm{pc})$ & $L^{\prime}-M^{\prime}$ & $K \mathrm{~s}-L^{\prime}$ \\
\hline Source IR-CN & 0.1 & 7 & 1.5 & 1.7 \\
Source IR-CS & 0.1 & 7 & 1.4 & 2.0 \\
Struct. IR-1b north & 0.15 & 10 & 1.9 & 3.2 \\
Struct. IR-1b south & 0.15 & 10 & 1.4 & 3.1 \\
IR-6 & 0.15 & 10 & 1.7 & 2.5 \\
Knot IR-1 & 0.08 & 6 & 2.2 & $>3.0$ \\
Knot IR-2 & 0.08 & 6 & 2.2 & - \\
Knot IR-3 & 0.08 & 6 & 2.0 & - \\
Knot IR-4 & 0.08 & 6 & 2.1 & - \\
\hline
\end{tabular}

put an upper limit of a $10 \%$ uncertainty on our photometric measurements.

Moreover, the values we deduced are consistent, at larger scales, with values found in previous studies (Rouan et al. 1998 for the A.O. Ks band imaging and Marco \& Brooks 2003 for $L^{\prime}$ imaging). The $K \mathrm{~s}$ band values for IR knots and spots have been estimated on coronagraphic observations reported in GRB04.

There is no proven method to perform absolute photometry on deconvolved data. Nevertheless, one can estimate a relative photometry assuming that the color ratios of the central part are unchanged after deconvolution. So, we calculated color indices from data after deconvolution. Under this hypothesis, the $L^{\prime}-M^{\prime}$ values found for previously identified structures are in good agreement with most of the values before deconvolution, and so give some confidence in the estimated flux of newly found structures (IR-CN and IR-CS) and sub-structures in IR-1b. In the case of $K \mathrm{~s}-L^{\prime}$, the deconvolved color indices disagree with non-deconvolved ones in most regions, probably because at $K \mathrm{~s}$, most of the sub-structuren was fainter and embedded in the diffuse background. The deconvolution process could fail to simultaneously restore a correct shape and photometry in regions dominated by diffuse emission. This does not call into question the morphology found at $K \mathrm{~s}$, which superimposed nicely with the ones found at other wavelength, but no safe interpretation of the deconvolved photometry can be done.

\subsection{General registration with maps at other wavelengths}

As shown in RLG04, the NaCo images can be well registered with maps obtained at other wavelength. Here, the deconvolution makes all structures clearer, suppressing the airy rings, and reducing the scattered light from the nucleus. The superimposition with maps at other wavelengths can be done more accurately, at the level of a few tens of mas. Following Galliano et al. (2003) the nuclear source in the infrared can be superimposed on the identified nuclear source in the radio domain (source $\mathrm{S} 1$ ). Radio source $\mathrm{C}$ is then coincident with our source IR-1b (Fig. 4d), and the radio jet is bracketed by the wave-like series of elongated knots at N.E., and the structure IR-5.

The structures seen in deconvolved mid-IR data of Bock et al. (2000) are very similar to those observed with $\mathrm{NaCo}$ (RLG04). However, our deconvolved images show a more complex sub-structure when superimposed, especially in the structure named the tongue (Fig. 4f).

Deconvolved images can be very well registered with UV clouds, identified first by Evans et al. (1991), on HST UV [O III] images of Capetti et al. (1997) and narrow band $\mathrm{H} \alpha$ images of Thompson et al. (2001). Assuming that the UV polarization center, located at the lower end of cloud B (Kishimoto 1999), is coincident with our IR quasi-pointlike central source, as first argued in RLG04, we find counterparts for clouds C, D, $\mathrm{E}$ in our $L^{\prime}$ and $M^{\prime}$ images. Note that the central IR core has also been identified as the near-IR polarization center within $1^{\prime \prime}$ (Simpson et al. 2002). One can also notice the remarkable resemblance between structure IR-5 in the deconvolved $M^{\prime}$ image and the HST cloud F observed in [OIII] and $\mathrm{H} \alpha$, both exhibiting the same shape with two components. This registration scheme, especially with HST clouds, is in agreement with the one proposed by Bock et al. (2000). All these registrations are only based on morphological resemblance. A fine resolution multi-wavelength study within $1^{\prime \prime}$ is now achievable thanks to A.O. observations in the near-IR coupled with deconvolution.

\subsection{Morphology}

\subsubsection{The compact core}

On non-deconvolved images, the prominent compact nuclear source was partly resolved along the North-South direction at $K$ s, as shown in the $K$ s profiles of RLG04. This central source is also marginally resolved in the $L^{\prime}$ band and totally unresolved at $M^{\prime}$. The apparent size of this nucleus for each band is reported in Table 3.

After deconvolution, the central source does not exhibit exactly the same morphology from $K$ s to $M^{\prime}$ bands. At all bands, a quasi-pointlike central source, linked to a northern spot named IR-CN is seen. This can explain the asymmetric profiles of the central source found at $K$ s and $L^{\prime}$. However, at 

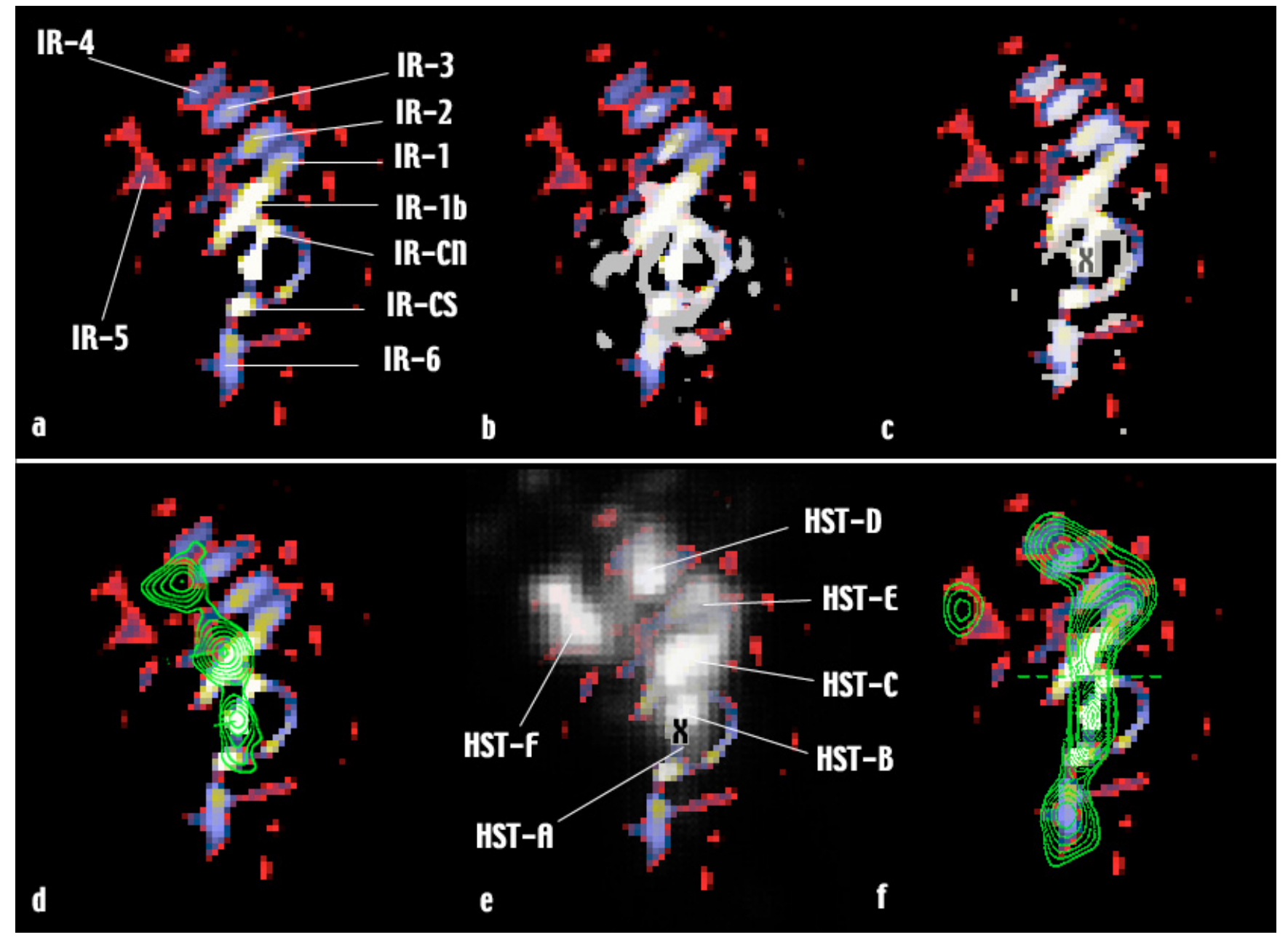

Fig. 4. a)-Name given to identified structures in these lines. b)-Superposition of $K$ s (white) and $M^{\prime}$ deconvolved images. c)-Superposition of $L^{\prime}$ (white) and $M^{\prime}$ deconvolved images. The black cross indicates the location of the central source. d)-Radio map (green contours) from Gallimore et al. (2001) over deconvolved $M^{\prime}$. e)-HST [OIII] observations (white) from Capetti et al. (1997) over deconvolved $M^{\prime}$. The black cross marks the location of the IR central source. f)-mid-IR deconvolved contours from Bock et al. (2000) (green) over deconvolved $M^{\prime}$.

Table 3. FWHM of the core at each wavelength before (undec) and after (dec) deconvolution, compared to the size of the PSF and the diffraction limit.

\begin{tabular}{ccccc}
\hline \hline Band & $F W H M_{\text {undec }}$ & PSF & Diff. limit & $F W H M_{\text {dec }}$ \\
\hline$K \mathrm{~s}(\mathrm{~N}-\mathrm{S})$ & $0.08^{\prime \prime}$ & $0.06^{\prime \prime}$ & $0.056^{\prime \prime}$ & $30 \operatorname{mas}(2.1 \mathrm{pc})$ \\
$K \mathrm{~s}(\mathrm{E}-\mathrm{W})$ & $0.065^{\prime \prime}$ & - & - & $<15 \mathrm{mas}(1.2 \mathrm{pc})$ \\
$L^{\prime}$ & $0.11^{\prime \prime}$ & $0.10^{\prime \prime}$ & $0.098^{\prime \prime}$ & $<0.05^{\prime \prime}(3.5 \mathrm{pc})$ \\
$M^{\prime}$ & $0.12^{\prime \prime}$ & $0.12^{\prime \prime}$ & $0.12^{\prime \prime}$ & $<0.05^{\prime \prime}$ \\
\hline
\end{tabular}

$K$ s, the source clearly has an elongated shape along the same PA $\left(-16^{\circ}\right)$, as described in the $K$ band bi-spectrum speckle interferometric observations of Weigelt et al. (2004). The size of the elongation $(2.1 \mathrm{pc})$ found after deconvolution, discussed in Sect. 4, is nevertheless smaller than the one they derived. At other wavelengths the central source, despite its link to the northern spot, appears rather circular in the inner $5.5 \mathrm{pc}$. We consider this value as an upper limit for its size of the core in these bands.

On the other side, $0.2^{\prime \prime}$ south of the nucleus, another spot appears in all bands. We called it IR-CS. This spot is not obviously linked to the central source and seems extended at all wavelength. The three sources (IR-CN, IR-CS and the central source) are aligned along PA $\sim-20^{\circ}$. Source IR-CN is coincident with part of HST cloud B but not with any radio structure. The Spot IR-CS is very close to HST cloud A as well as the radio source $\mathrm{S} 2$. These spots could trace the dusty cores of gas clouds orbiting the central engine, screening external regions in that direction from the central activity. The magnitude difference after deconvolution between these spots and the central source is about 3 at $M^{\prime}$ and $L^{\prime}$ and 2 at $K$ s and, in all bands, the flux ratio between the two spots is close to unity. This is consistent with dusty clouds heated directly by the central XUV source since, with a simple radiative transfer model, we can reproduce a $600 \mathrm{~K}$ dust temperature. These clouds would be in the innermost part of the NLR, where dust is close to sublimation.

\subsubsection{Knot IR-6}

On the south part of the nucleus, only a small tail appears $0.35^{\prime \prime}$ of the nucleus. This patch is elongated N-S $\left(0.22^{\prime \prime}\right)$ and exhibits 
substructures while it is unresolved in the E-W direction. Its upper part is aligned with the three previous sources. This structure was first identified in mid-IR by Bock et al. (2000), who found an elongated spot south of the central source. Neither HST cloud nor radio source can be identified at this location. The lack of intense UV emission may be due to screening by the inclined galactic plane of NGC 1068, the southern NLR clouds being more conspicuous in the IR (Thompson et al. 2001). Its spectral energy distribution in the mid-IR is comparable to the tongue (Bock et al. 2000), observed north of the nucleus at about the same distance, so they might be of the same nature. However its $L^{\prime}-M$ color is redder than the one found in the northern structures. Obscuration by dust could be responsible for this effect.

\subsubsection{Micro-spiral, bar-like or jet induced structure IR-1b}

An elongated structure identified as a micro-spiral arm in a previous paper (RLG04) is detected in all deconvolved images, bending north of the nucleus. It looks like a spiral arm in $K$ s and $L^{\prime}$, but is straighter and more elongated in the $M^{\prime}$ band. This feature was first identified by Rouan et al. (1998) and depicted as a possible micro-spiral arm. Their deconvolved K-band image revealed a S-shaped extended structure, including a central bar and two micro-spiral arms crossing the nucleus. Mid-IR deconvolved data of Bock et al. (2000) showed a more linear structure aligned along PA $=0$, with a shorter southern part, resembling a large spot (our spot IR-6), linked to the northern structure. The NaCo data revealed two components in the northern part. We identified in RLG04 a feature which seemed to bend north-east, a possible trace of a northern micro-spiral arm. This structure that we named IR-1b ends with knot IR-1. It is coincident with HST cloud C which is known to be at the interface between the radio jet and a giant molecular cloud, as demonstrated in Gallimore et al. (1996a). This radio source is known for its jet mega-masers that Gallimore et al. (2001) suggested to be powered by X-ray emission induced by the shock. However Kraemer \& Crenshaw (2000b) argued that most of its UV continuum is nuclear scattered light and cloud $\mathrm{C}$ seems mostly photoionized. In addition, Cecil et al. (2002) showed that, because of the UV line ratio, the deflection of the jet is not clearly connected to the excitation of the cloud in the UV, even if the broadening of lines is consistent with shocks. This region appears also polarized in the near IR (Simpson et al. 2002). Considering the high UV and X-ray radiation field and the presence of high velocity shocks, the survival of the dust in this region is an issue.

The straight shape of this structure in the near-IR, especially at $M^{\prime}$, is also a challenging question. 3-D simulations of jet-cloud interaction in YSOs (de Gouveia Dal Pino 1999) conversely exhibit a clumpy distribution of the emission which is hard to compare to IR-1b seen in $M^{\prime}$; however, in $K$ s and $L^{\prime}$, this structure appears more disturbed as expected in the case of a jet-cloud interaction. Patchy obscuration by dust could also be responsible for such differences and we thus favor the interpretation of a dusty cloud interacting with the jet.

\subsubsection{Jet-induced structures IR-1 to IR-5}

The fourth remarkable feature is a "wave-like" series of four (probably five given the deconvolved $M^{\prime}$ band) elongated knots that begin at $0.4^{\prime \prime}$ north of the nucleus (named knot IR-1 to IR4 in GRB04) and developing along PA $=47^{\circ}$ for about $50 \mathrm{pc}$, very well resolved in one direction after deconvolution. These knots are elongated about 10pc each, unresolved $(<4 \mathrm{pc})$ in the other direction and perfectly parallel along $\mathrm{PA}=-45^{\circ}$. A peculiar structure (resembling a crab pincer) that we named IR-5 located $0.7^{\prime \prime}$ north-east along $\mathrm{PA}=47^{\circ}$, appears only in $M^{\prime}$ and is sub-structured into two components, with an extension along the same direction as knots IR-1 to IR-4. Using deconvolution, structures are better defined than in the previous study.

What could be at the origin of this surprising pattern?

A first mechanism that can be invoked to explain the quasiperiodic shape of this wave-like group of knots over such a distance is periodic winds, blowing from the central engine (Elvis 2000). However the knots would be expected to be arranged as concentric arcs centered on the nucleus, which is not verified.

Another process could be jet-ISM interaction. We first note that HST UV-visible, mid-IR and our near-IR images present a cloud-free region, precisely where the radio jet propagates, surrounded by a more clumpy medium, composed of the dense aligned clouds. The structured arrangement of IR knots along the jet suggests hydrodynamic effects induced by the latter. Several numerical simulations have been developed aiming at describing the interaction of the jet with the ISM.

- Saxton et al. (2002) have obtained ring-like radio emitting knots surrounding a jet passing through a dense medium. This structures are reminiscent of what we observe in nearIR. However the scales are very different and no periodic pattern is evident. Moreover, NGC 1068 does not show any radio feature of that type.

- Micono et al. (2000, 1998a,b) have developed a general hydrodynamic model for radiative jets, especially suited to the stellar case, where Kelvin-Helmotz instabilities play the basic role. In their simulations, interaction of the jet with the ISM induces either regular structures but with a bowshock appearance or a fully turbulent morphology. Thus the comparison with our images is not convincing.

- The model developed by Steffen et al. (1997) is more relevant to the NGC 1068 case, because it deals with clouds in the NLR of an AGN. Their simulations produce dense clumps in a cocoon surrounding the jet, but they appear more randomly distributed than the four IR knots we detected.

Even if, as far as we know, no simulation seems to be able to reproduce the regular pattern, we still tend to favor a hydrodynamic effect. Two of these knots are also perfectly coincident with observed HST UV clouds D and E, following our registration scheme, and structure IR-5 is superimposed on cloud F. However, the whole set of HST clouds does not exhibit the same structured distribution.

We exclude the possibility that dust is intrinsically mixed with ionized gas in the UV clouds, since the IR knots are not resolved in the direction parallel to the jet, while the UV sources 
appear diffuse. We rather believe that we are observing a class of objects that would be made of a dense dusty core surrounded by an ionized gaseous envelope. The patchy and more severe extinction by dust in the UV-visible could explain the non regular shape of the UV emission.

There is also a correlation between the presence of these knots and the presence of knots in the radio jet, which reinforces the interpretation of jet-induced structures. However why the periodicity of the near-IR knots is not reproduced within the radio jet is an open question.

Complementary information (velocity, spectroscopy, etc.) on these very peculiar structures is required to understand their nature.

\subsubsection{Other structures}

Several other spots are found in the deconvolved images: one spot $0.3^{\prime \prime}$ south-west of the nucleus, another one is located $0.43^{\prime \prime}$ north-west, and a last one $0.75^{\prime \prime}$ north of the nucleus. The latter was first identified on coronagraphic images of GRB04 and named spot N2. West of the nucleus, an arc is identified in $L^{\prime}$ and $M^{\prime}$ band images and partially detected in $K$ s. The arc is well superimposed in all bands favoring the conclusion that this strange shape is not a residual Airy pattern. Nevertheless, this feature has not been detected on the coronagraphic $K \mathrm{~s} \mathrm{im}-$ age, probably because of the coronagraphic mask pattern, so we cannot exclude the possibility that this arc can be an artifact of deconvolution.

\section{Comparison to numerical models}

Since no $\mathrm{H}_{2}$ emission is detected within a $0.5^{\prime \prime}$ radius around the central engine (Alloin et al. 2001; Galliano \& Alloin 2002; Gratadour et al. 2003), and there is no evidence of high stellar activity in its surroundings (Marco \& Brooks 2003), we will assume in the following that most of the near-IR emission is due to dust, and we will explore possible excitation mechanisms to explain the color indices found.

\subsection{Temperatures}

If we assume classical grains, for instance silicate grains, heated by the central engine and no screening between the two, the temperature of grains at a given distance $r$ to the nucleus is approximated by:

$$
T=1650 \times\left(\frac{L_{\mathrm{uv}, 46}}{r_{\mathrm{pc}}^{2}}\right)^{\frac{1}{5.6}} \mathrm{~K}
$$

where $L_{\mathrm{uv}, 46}$ is the luminosity of the exciting source in units of $10^{46} \mathrm{erg} \mathrm{s}^{-1}$ (Barvainis 1987). It puts an upper limit on the temperature induced by this excitation mechanism. The temperatures found with this simple model are reported in Table 4. We assumed a UV luminosity of $1.5 \times 10^{45} \mathrm{erg} \mathrm{s}^{-1}$ for the central source of NGC 1068, a value that allowed us to reproduce the central $K$ band flux and continuum in a previous study (Gratadour et al. 2003). The color temperatures deduced from deconvolved data for structure IR-CN and IR-CS are in good
Table 4. Temperatures deduced from values of the color indices in each identified region, before and after deconvolution, compared to values found considering the distance to the nucleus and no screening. Only the deconvolved $L^{\prime}-M^{\prime}$ is considered because in good agreement. Classical Black Bodies are assumed to evaluate temperatures.

\begin{tabular}{ccccc}
\hline \hline Position & $T_{\text {undec }}$ & $T_{\text {dec }}$ & Dist $(\mathrm{pc})$ & $T_{\text {model }}$ \\
\hline Source IR-CN & - & 600 & 10 & 520 \\
Source IR-CS & - & 600 & 10 & 520 \\
Struct. IR-1b north & 650 & 600 & 25 & 375 \\
south & 650 & 600 & 25 & 375 \\
IR-6 & 600 & 500 & 25 & 375 \\
Knot IR-1 & 550 & 450 & 30 & 350 \\
Knot IR-2 & 500 & 450 & 40 & 315 \\
Knot IR-3 & 500 & 450 & 55 & 280 \\
Knot IR-4 & 450 & 450 & 65 & 265 \\
\hline
\end{tabular}

agreement with this simple model. For the other structures, the predicted temperatures are far too low and we need another mechanism to explain the high temperatures in region IR-1b and IR-2 to IR-4 with in addition practically no variation with distance.

\subsection{Shocks}

Shocks might be a mechanism able to provide an efficient local heating. Indeed, it is commonly assumed that radio source $\mathrm{C}$ traces the deflection of the jet, probably by a giant molecular cloud. Following Villar-Martín et al. (2001), dust grains can survive in highly ionized and shocked regions if, for instance, NLR clouds consist of an external hot shell of ionized gas, with a cooler and denser dusty core.

If we assume a shocked cloud of density $n$, the induced UV luminosity was given by Dopita \& Sutherland (1996) to be:

$L_{\mathrm{uv}, \text { shock }}=1.1 \times 10^{-3}\left(\frac{v_{\mathrm{s}}}{100 \mathrm{~km} \mathrm{~s}^{-1}}\right)^{3.04} \frac{n}{\mathrm{~cm}^{-3}} \operatorname{erg~cm}^{-2} \mathrm{~s}^{-1}$.

The density of the cloud responsible for the deflection of the jet was estimated by Gallimore et al. (1996a) to be at least of about $100 \mathrm{~cm}^{-3}$, considering the radio jet properties, a value consistent with densities of giant molecular clouds found in the galactic plane. Concerning the shock velocity, Axon et al. (1998) found that the ionization conditions and the velocity structures are consistent with shocks of velocity of at least $700 \mathrm{~km} \mathrm{~s}^{-1}$. Assuming these initial conditions, and a size of about $10 \times 10 \mathrm{pc}$ for HST cloud C, the UV flux induced solely by the shock generated by the interaction of the jet with the cloud should be of about $2 \times 10^{40} \mathrm{erg} \mathrm{s}^{-1}$. If we find an upper value, considering a dense cloud $\left(n=200 \mathrm{~cm}^{-3}\right)$ and a faster shock $\left(v_{\mathrm{s}}=1000 \mathrm{~km} \mathrm{~s}^{-1}\right)$ the shock-generated UV flux becomes: $L_{\mathrm{uv}, \text { shock }}=1.2 \times 10^{41} \mathrm{erg} \mathrm{s}^{-1}$. This is an order of magnitude less than the flux received from the central source at this distance $\left(2.4 \times 10^{42} \mathrm{erg} \mathrm{s}^{-1}\right)$. Shocks alone are thus not able to explain the high color temperatures found in knots IR-1 and IR-2. For IR-1b, as previously argued in Sect. 3, spectroscopic studies in the UV have shown that most of the UV emission from cloud $\mathrm{C}$ comes from scattered nuclear light rather than from shocks. For structure IR-3, IR-4 and IR-5, Gallimore et al. (1996a) found no evidence for the formation of a shock front. 
So, if classical dust grains are considered, it does not seem that shocks are able to reproduce the high color temperatures of knots IR-1 to IR-4.

\subsection{The VSG hypothesis}

Another way to tackle this issue is to explore the nature of the dust. In our previous paper (RLG04 and RGB04) we proposed that transient heating of very small dust grains (VSG), possibly nano-diamonds, could be responsible for these high color temperatures. Here, we explore this hypothesis in more details.

\subsubsection{General model for carbonaceous VSG}

First proposed to explain the bump at $220 \mu \mathrm{m}$ in interstellar IR emission, transient heating of VSG could be responsible for $40 \%$ of the energy absorbed by dust in the ISM (Desert et al. 1990). It could also be responsible for the unidentified midIR emission bands (Guillois et al. 1999). Thus, it represents a very good candidate to explain the high dust temperature in UV-irradiated regions such as knots IR-1 to IR-4.

We consider here the general case of a single UV photon heating a nano-grain. In order to estimate its maximal temperature as well as the the flux ratio between near-IR bands during the cooling phase, we need to estimate the heat capacity of a VSG. It can be approximated by a Debye law at low temperature and a constant (Dulong and Petit model) at high temperature. This can be conveniently written as:

$C=\frac{\left(C_{0} \frac{T}{T_{0}}\right)^{3}}{1+\frac{C_{0}}{C_{1}}\left(\frac{T}{T_{0}}\right)^{3}}$

where $T_{0}$ is the Debye temperature of the dust species and $C_{0}$ and $C_{1}$ coefficients depend on the characteristics of the grains. We took

$C_{0}=2.38 \times 10^{4} a_{\mathrm{cm}}^{3}\left(\frac{T}{T_{0}}\right)^{3}$

and

$C_{1}=30.5 \times a_{\mathrm{cm}}^{3}$

(units $\mathrm{JK}^{-1}$ ), assuming simple carbonaceous nano-grains. Following Jones \& d'Hendecourt (2000), we adopt a Debye temperature of $2000 \mathrm{~K}$, and we obtain a temperature evolution with grain radius and incident photon energy in good agreement with the one they obtained with a more sophisticated dust grain model.

When the photon is absorbed by the grain, it heats it at a maximum temperature which depends on the size of the grain and the photon energy. The grain then cools by re-emitting this energy, mostly in the IR. Assuming a blackbody emission for the grain, we can deduce the total flux emitted by a cooling grain of equivalent radius:

$F(\lambda)=\int \pi B_{\lambda}[T(t)] \times 4 \pi a^{2} \mathrm{~d} t$

where $T(t)$ is the solution of the energy conservation equation:

$C d T=4 \pi a^{2} \sigma T^{4} \mathrm{~d} t$.

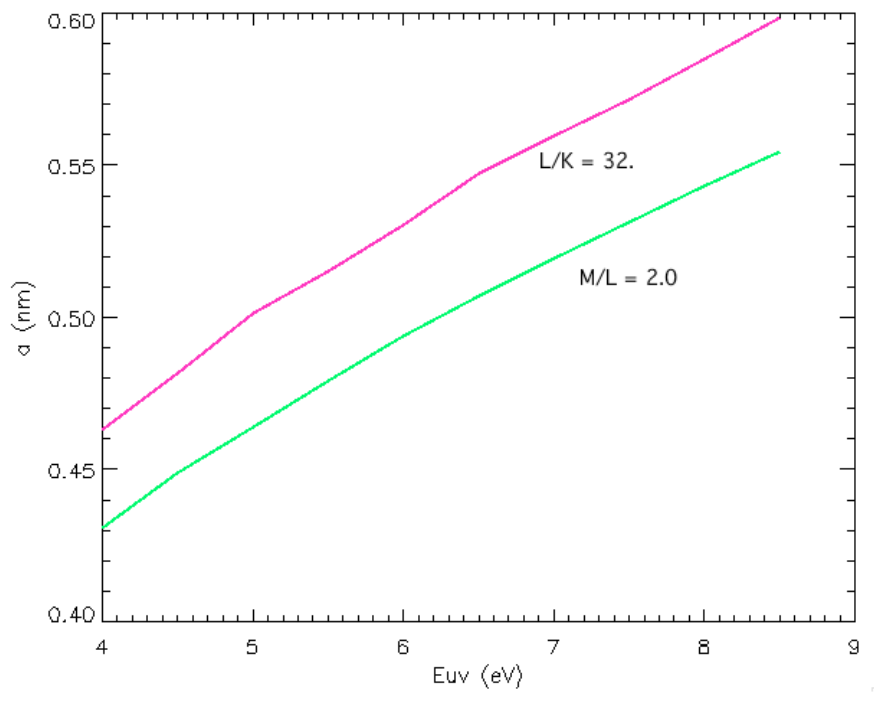

Fig. 5. Grain radius and incident energy thhat reproduce the $K$ s-Lp and Lp-M observed on our data. The ratios are indicated in flux, not in magnitude. The mean solution which would best reproduce the two color ratio is indicated with the dark cross.

We can thus obtain the flux ratio between near-IR bands for the cooling of a carbonaceous VSG. We tried to reproduce the $L^{\prime}-M$ and $K s-L^{\prime}$ colors found for knot IR-3, for instance with a variety of grain sizes and photon energies. We plot in Fig. 5 the solutions found for both of the two color ratios.

We note: a) within a plausible range of photon energy, the grain size does not vary by a large amount $(\approx 25 \%)$; b) the two solutions found are rather close and the best fit to the data may be in between, given the uncertainties. Globally, a mean solution which would best reproduce the color ratio found in knots IR-1 to IR-5 would be $6 \mathrm{eV}$ UV photons and grains with a radius of about $0.5 \mathrm{~nm}$.

\subsubsection{Nano-diamonds: interesting candidates}

In RLG04 we proposed that nano-diamonds could be the major component of the VSG population in those knots. Laboratory experiments have shown that the temperature of a nanodiamond can increase to very high values (up to $1000 \mathrm{~K}$ ) when absorbing one UV photon (Jones \& d'Hendecourt 2000). Nano-diamonds are a very stable and resilient species that can be formed by strong UV radiation on graphitic or anamorphous carbonaceous grains by chemical vapor deposition (Hill et al. 1998). They can also form in shocks, as indicated by their discovery in meteorites subjected to high pressures (Lewis et al. 1987). Laboratory experiments show that most of the emission appears in emission bands given the very low value of $Q_{\text {abs }}$ at other wavelengths (diamond is well-known to be transparent in the IR), but, on the other hand, the presence of emission bands at $3.43 \mu \mathrm{m}$ and $3.53 \mu \mathrm{m}$ is clear for large diamond particles only and would not appear for very small ones (Jones et al. 2004), so that we no longer predict the presence of such spectral features in NGC 1068 


\subsection{Dust in the central core}

Both deconvolution methods show an increase in the apparent size of the central source with wavelength which is consistent with a simple physical model, assuming that the hottest dust is seen at shortest wavelengths. However, the source was only clearly resolved along N-S at $K$ s. Discussion about its actual physical size is thus reasonable only at this wavelength. Considering the L1-L2 method as the one that favors the highest intensity gradient in the object, the FWHM deduced from images deconvolved with this method should thus be considered as an upper limit on the size of the central core.

The near-IR colors of the central source found before deconvolution are consistent with dust at about $900 \mathrm{~K}$. This is in good agreement with previous conclusions based on spectroscopic observations (Gratadour et al. 2003). The central source seen at $2.2 \mu \mathrm{m}$ may represent the outer surface of a dust sublimation cavity around the central engine. The size of this cavity depends on the visible-UV luminosity of the central engine: $r_{\text {cav }}=1.3 \times L_{46}^{1 / 2}$ in pc, where $L_{46}^{1 / 2}$ is the visible-UV luminosity in units of $10^{46} \mathrm{erg} \mathrm{s}^{-1}$ according to Barvainis (1987). This leads to $0.5 \mathrm{pc}$ in the case of NGC 1068, assuming the same visible-UV luminosity as previously used.

When fitting the deconvolved central source by an elliptical 2-D Gaussian distribution (see Fig. 6), we find a FWHM of 30 mas, i.e $2.2 \mathrm{pc}$, at $K$ s along the major extension $\left(-16^{\circ}\right)$ and lower than 1. pc (13 mas) in the perpendicular direction (as expected since the source is unresolved in the E-W direction). This result is not in conflict with the interferometric observations of Wittkowski et al. (2004) with the VLTI instrument VINCI who found a size of 5 mas (i.e. 0.4 pc) for the central source along $\mathrm{PA}=45^{\circ}$ (i.e. the direction along which we do not resolve the source). We find also a circular 2-D Gaussian of $5.5 \mathrm{pc} F W H M$ in $L^{\prime}$ and $M^{\prime}$, but, as the source was not clearly resolved in these bands, this result must be considered as an upper value.

This kind of behavior, with an N-S elongation in the thermal IR, is indeed predicted by numerical models. The one that we developed to fit $K$ band continuum (Gratadour et al. 2003) reproduces such effects. We thus computed high resolution (2 mas/pixel) images with this model, using the set of parameters that allowed us to reproduce the central $\mathrm{K}$ continuum in terms of slope as well as in terms of flux, to compare with our near-IR images: i.e a torus inclined $22^{\circ}$ with regard to the line of sight, composed of silicate grains and a total bolometric luminosity of $1.5 \times 10^{45} \mathrm{erg} \mathrm{s}^{-1}$ in the visible-UV range for the central exciting source. The results are very similar at 2.2 and $4 \mu \mathrm{m}$ and show an emission, dominated by a central core, extending along the N-S direction. As shown in Fig. 6, the morphology of the source is totally different at $10 \mu \mathrm{m}$, where a more complex emission distribution appears in the central part and cannot obviously be fitted either by a single Gaussian component or by two Gaussian, as in the $2-5 \mu \mathrm{m}$ range. Our result is thus not directly comparable to the results of Jaffe et al. (2004) who fitted their interferometric data with two Gaussian components.

Nevertheless, the size of the N-S extension given by our model $(F W H M \approx 0.7 \mathrm{pc} \mathrm{N}-\mathrm{S}$ at $2.2 \mu \mathrm{m})$ is smaller than the one fitted on our $K$ s data, even if the general trend of a N-S extended source is clearly reproduced.

So, the combined interpretation of both photometric and morphological properties of the central source is not fully consistent. The over-simple model of a doughnut-like obscuring torus with a sublimation cavity is probably not sufficient to explain the more complex reality. Other models are needed to fully describe the central emission in the thermal IR.

For the size of this obscuring structure, no constraint on its outer scale is brought by our data. On the other hand, we can conclude that its internal wall is at a parsec scale, as shown by the $K \mathrm{~s}$ band image. Such a size is coherent, for instance, with the NaCo observations of Circinus, another archetypal type II nucleus (Prieto et al. 2004).

\section{Conclusion}

After careful image processing and reliable deconvolution, near-IR NaCo observations of NGC 1068 , from $K$ s to $M^{\prime}$ band, show a wealth of details and reveals a very complex dust structure around this AGN. The main results can be summarized as follows:

- The overall picture in the near-IR suggest the shaping of the NLR by the passage of the jet. At the location of radio source $\mathrm{C}$, a very peculiar elongated emission is found, appearing very straight in $M^{\prime}$, while more clumpy and bending at $K \mathrm{~s}$ and $L^{\prime}$. This difference may stem from patchy obscuration by dust. This structure probably trace the cloud interacting with the jet but its shape is unclear.

- Four elongated knots (IR-1 to IR-4), not resolved in one direction, quasi-periodically dispatched along the axis of the jet and bracketing it with another complex structure (IR-6) are shown. Their highly regular distribution is unexplained since no numerical simulations of the jet interacting with clouds or ISM is able to reproduce their regular alignment nor their elongation perpendicular to the direction of the jet. However, we favor an interpretation in terms of hydrodynamical instabilities due to interaction of the jet with its environment, given the close correlation between the structure alignment and the jet direction.

- Comparison to a simple model demonstrates that the very high color temperatures found in these structures cannot be produced by excitation induced by shock generated UV-X rays. On the other hand, transient heating of VSG of the size of about $0.6 \mathrm{~nm}$ is able to produce the high temperatures, and nano-diamonds are a very interesting candidate given their resistance and their formation mechanisms. However, their IR emissivity is not known and this requires further work.

- We find two new spots symmetrically disposed North-West and South-East of the central source, along PA $=-20^{\circ}$. They have similar magnitudes and a $\Delta_{\text {mag }}$ of 2 with the central source, which is consistent with dust at $600 \mathrm{~K}$, i.e. what is expected at this distance $(20 \mathrm{pc})$ in dusty clouds directly heated by the central engine.

- The central source is elongated along the same axis and resolved in $K$ s, with a $F W H M$ of $2.2 \mathrm{pc}$ in $K \mathrm{~s}$ at $\mathrm{PA}=-16^{\circ}$ 


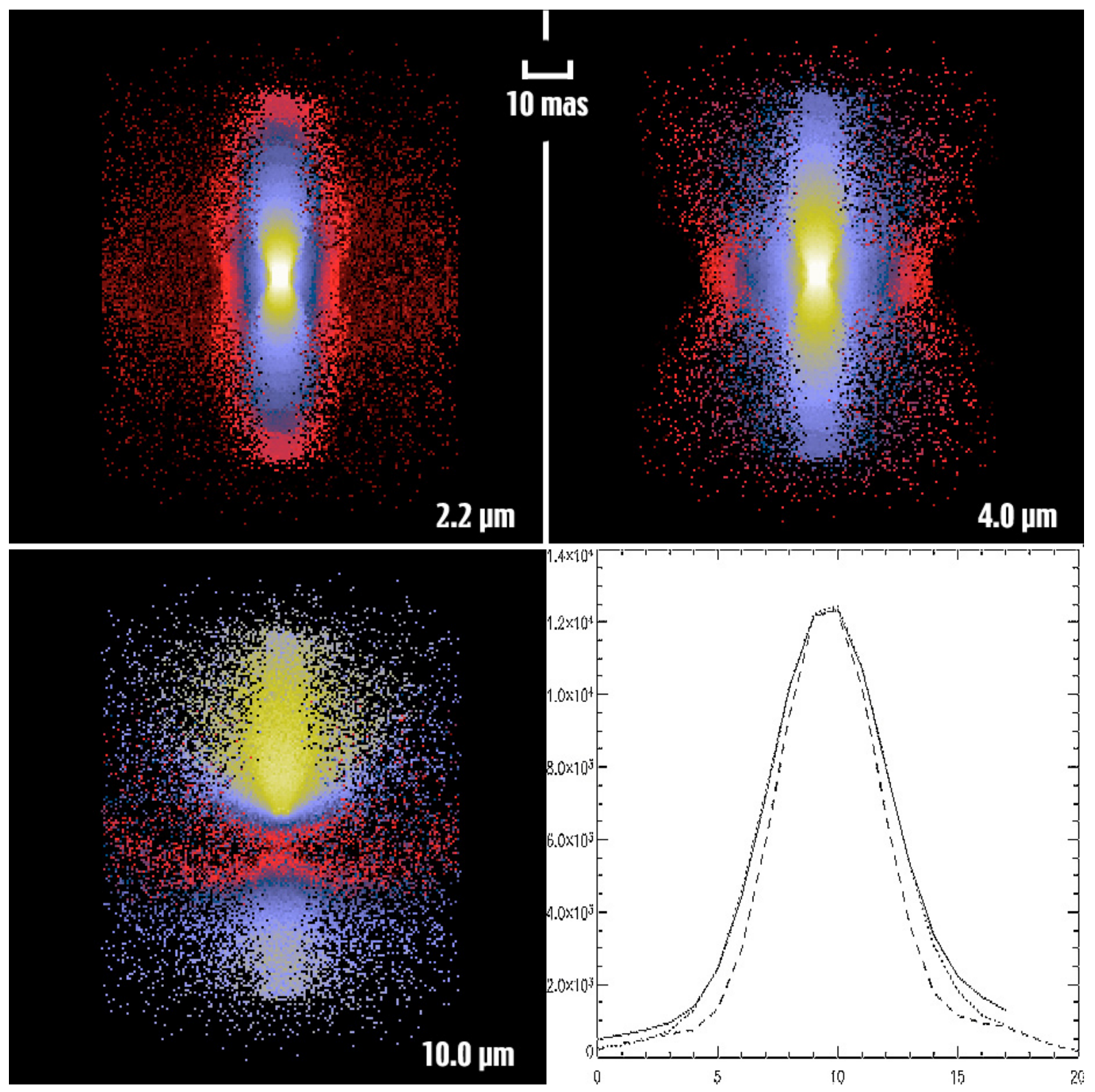

Fig. 6. Images obtained with our radiative transfer model at 2.2, 4.0 and $10.0 \mu \mathrm{m}$, and the N-S profile on the $K$ s image of NGC 1068 (solid line) compared to the N-S profile of the image obtained by convolving the elliptical 2-D Gaussian fitted on deconvolved $K \mathrm{~s}$ image by the PSF (dotted line) and the isotropic profile of the PSF (dashed line).

and less than $1 \mathrm{pc}$ in the perpendicular direction. We also put an upper limit of $5.5 \mathrm{pc}$ on the source $F W H M$ in $L^{\prime}$ and $M^{\prime}$. This elongated shape is reproduced with a radiative transfer model tuned to reproduce previous spectroscopic data. Nevertheless, the size found with this model is smaller than the one deduced from deconvolved images. This could call into question the relevance of the doughnut torus paradigm which is certainly an over-simple description of a complex reality, as testified, for instance, by our discovery of waves bracketing the jet.

Through careful image processing we extracted new information. Nevertheless, this near-IR dissection of the nucleus of NGC 1068 done with the best single-dish resolution achievable in the near-IR is limited to the study of the NLR.
What new observations are needed? The unambiguous description of the BLR and the dust sublimation cavity of this object will soon be reached by interferometric techniques, for instance with the new VLTI instruments such as AMBER, especially when multi-baseline observations can ensure image reconstruction. The emission spectrum of transiently heated particles is different to the standard black body distribution (Desert et al. 1990), and spectra from 2 to $5 \mu \mathrm{m}$ of the IR knots could help to discriminate between the VSG component proposed here and classical grains. Narrow band imaging in coronal lines and recombination hydrogen lines would improve the contrast and test the spatial correlation between gas and dust. Polarization in the IR could also help to discriminate between scattered nuclear light from the AGN and intrinsic emission. 


\section{Appendix A: Classical deconvolution with a PSD type regularization}

The deconvolution process is based on a maximum a posteriori estimation of the object, meaning the minimization of a compound criterion:

$J(\mathbf{o})=J_{i}(\mathbf{o})+J_{o}(\mathbf{o})$.

The first term of this criterion is a measure of the fidelity to the data, and the second term, a regularization or penalty term.

Concerning the object prior, assuming a Gaussian prior probability distribution leads to deriving its expression in the Fourier domain from the computation of its Power Spectral Density (PSD). The regularization term is thus written as (Conan et al. 1998):

$J_{o}(\mathbf{o})=\mu \frac{1}{2} \sum_{f} \frac{\left|\tilde{\mathbf{o}}(f)-\tilde{\mathbf{o}}_{\mathbf{m}}(f)\right|^{2}}{S_{o}(f)}$,

where $\tilde{\mathbf{o}}_{\mathbf{m}}=E[\tilde{\mathbf{o}}]$ is the mean Fourier transform, and $S_{o}=$ $E\left[\left|\tilde{\mathbf{o}}(f)-\tilde{\mathbf{o}}_{\mathbf{m}}(f)\right|^{2}\right]$ is the object PSD. The weight of the regularization term in the compound criterion should be adjusted according to the noise level in the image.

\section{A.1. Unsupervised estimation of the object and noise PSDs}

We choose the following parametric model for the object PSD $S_{o}$ (Conan et al. 1998):

$S_{o}(f)=E\left[\left|\tilde{\mathbf{o}}(f)-\tilde{\mathbf{o}}_{m}(f)\right|^{2}\right]=K /\left[1+\left(f / f_{0}\right)^{p}\right]$.

This model has the advantage of being both sparse (few parameters) and correctly modeling the PSD of various types of scenes.

If the noise is assumed to be Gaussian and approximately stationary (the case of a background-limited noise with a uniform background, as in the thermal IR), there are four hyperparameters to be adjusted for the deconvolution: the noise variance $\sigma_{n}^{2}$ and the parameters of the object PSD, $\theta_{o}=\left\{K, f_{o}, p\right\}$.

The adjustment of $\theta_{o}=\left\{K, f_{o}, p\right\}$ can be made in a supervised way using physical considerations: $K$ is approximately given by the square of the number of photons in the image, $f_{o}$ is the inverse of the characteristic size of the object, while the power $p$ characterizes the regularity or smoothness of the object and is usually between 1 and 4 . This supervised adjustment has proved useful (Conan et al. 1998) but is necessarily approximate.

It is possible, in a Gaussian framework, to identify (or estimate) the hyper-parameters in an non supervised way by a Maximum Likelihood (ML) method, as recently proposed in the context of phase diversity (Blanc et al. 2003). If we denote by $\Theta=\left\{K, f_{o}, p, \sigma_{n}^{2}\right\}$ the set of hyper-parameters, the method consists of integrating the observed object out of the problem and finding the values of the parameters that maximize the likelihood of the data.

Because $\mathbf{o}$ and $\mathbf{n}$ are assumed to be Gaussian and independent, $\mathbf{i}$ is Gaussian as the sum of two independent Gaussian processes. Its probability density $p_{i}(\mathbf{i}, \Theta)$, viewed as a function of $\Theta$ for a given $\mathbf{i}$, is the data likelihood. The opposite of the data $\log$-likelihood $L(\Theta)$ can be written, with a minor approximation, as:

$L(\Theta)=\sum_{f}\left[\log \left(|\tilde{\mathbf{h}}(f)|^{2} S_{o}(f)+\sigma_{n}^{2}\right)+\frac{\left|\tilde{\imath}(f)-\tilde{\mathbf{h}}(f) \tilde{\mathbf{o}}_{m}(f)\right|^{2}}{|\tilde{\mathbf{h}}(f)|^{2} S_{o}(f)+\sigma_{n}^{2}}\right]$.

The identification consists of minimizing $L$ of Eq. (A.4) as a function of $\Theta$, i.e., four parameters. The gradient of $L$ can be computed analytically, so $L$ can be minimized quickly by a conjugate gradient method. Additionally, it is possible to reduce to three the number of unknowns by making the change of variables $\mu=\sigma_{n}^{2} / K$ and searching for $\Theta^{\prime}=\left\{K, f_{o}, p, \mu\right\}$ instead of $\Theta=\left\{K, f_{o}, p, \sigma_{n}^{2}\right\}$. Thus nulling the gradient of $L\left(\Theta^{\prime}\right)$ with respect to $K$ gives an analytical expression of $\hat{K}$ as a function of the current values of the three other parameters $f_{o}, p, \mu$, which speeds up convergence.

\section{References}

Alloin, D., Galliano, E., Cuby, J. G., et al. 2001, A\&A, 369, L33 Antonucci, R. R. J., \& Miller, J. S. 1985, ApJ, 297, 621

Axon, D. J., Marconi, A., Capetti, A., et al. 1998, ApJ, 496, L75

Barvainis, R. 1987, ApJ, 320, 537

Blanc, A., Mugnier, L. M., \& Idier, J. 2003, J. Opt. Soc. Am. A, 20

Bock, J. J., Neugebauer, G., Matthews, K., et al. 2000, AJ, 120, 2904

Capetti, A., Macchetto, F., Axon, D. J., Sparks, W. B., \& Boksenberg, A. 1995, ApJ, 452, L87

Capetti, A., Macchetto, F. D., \& Lattanzi, M. G. 1997, ApJ, 476, L67

Cecil, G., Dopita, M. A., Groves, B., et al. 2002, ApJ, 568, 627

Clénet, Y., Rouan, D., Gratadour, D., et al. 2005, A\&A, 439, L9

Conan, J.-M., Mugnier, L. M., Fusco, T., Michau, V., \& Rousset, G. 1998, Appl. Opt., 37, 4614

Crenshaw, D. M., \& Kraemer, S. B. 2000a, ApJ, 532, 247

Crenshaw, D. M., \& Kraemer, S. B. 2000b, ApJ, 532, L101

Davies, R. I., Sugai, H., \& Ward, M. J. 1998, MNRAS, 300, 388

de Gouveia Dal Pino, E. M. 1999, ApJ, 526, 862

Desert, F.-X., Boulanger, F., \& Puget, J. L. 1990, A\&A, 237, 215

Dopita, M. A., \& Sutherland, R. S. 1996, ApJS, 102, 161

Elvis, M. 2000, ApJ, 545, 63

Evans, I. N., Ford, H. C., Kinney, A. L., et al. 1991, ApJ, 369, L27

Fadda, D., Giuricin, G., Granato, G. L., \& Vecchies, D. 1998, ApJ, 496, 117

Fusco, T., Rousset, G., Rabaud, D., et al. 2004, Pure Appl. Opt., 6, 585

Galliano, E., \& Alloin, D. 2002, A\&A, 393, 43

Galliano, E., Alloin, D., Granato, G. L., \& Villar-Martín, M. 2003, A\&A, 412, 615

Gallimore, J. F., Baum, S. A., \& O’Dea, C. P. 1996a, ApJ, 464, 198

Gallimore, J. F., Baum, S. A., O’Dea, C. P., \& Pedlar, A. 1996b, ApJ, 458,136

Gallimore, J. F., Henkel, C., Baum, S. A., et al. 2001, ApJ, 556, 694

Gratadour, D., Clénet, Y., Rouan, D., Lai, O., \& Forveille, T. 2003, A\&A, 411, 335

Gratadour, D., Mugnier, L. M., \& Rouan, D. 2005a, A\&A, 443, 357

Gratadour, D., Rouan, D., Boccaletti, A., Riaud, P., \& Clénet, Y. 2005b, A\&A, 429, 433

Guillois, O., Ledoux, G., \& Reynaud, C. 1999, ApJ, 521, L133

Hill, H. G. M., Jones, A. P., \& D'Hendecourt, L. B. 1998, A\&A, 336, L41 
Jaffe, W., Meisenheimer, K., Röttgering, H. J. A., et al. 2004, Nature, 429, 47

Jones, A. P., \& d'Hendecourt, L. 2000, A\&A, 355, 1191

Jones, A. P., d'Hendecourt, L. B., Sheu, S.-Y., et al. 2004, A\&A, 416, 235

Kishimoto, M. 1999, ApJ, 518, 676

Kraemer, S. B., \& Crenshaw, D. M. 2000a, ApJ, 532, 256

Kraemer, S. B., \& Crenshaw, D. M. 2000b, ApJ, 544, 763

Krolik, J. H. 1999, Active galactic nuclei (Princeton Univ Press)

Lenzen, R., Hofmann, R., Bizenberger, P., \& Tusche, A. 1998, in Infrared Astronomical Instrumentation, ed. A. M. Fowler, Proc. SPIE, 3354, 606

Lewis, R. S., Ming, T., Wacker, J. F., Anders, E., \& Steel, E. 1987, Nature, 326, 160

Macchetto, F., Capetti, A., Sparks, W. B., Axon, D. J., \& Boksenberg, A. 1994, ApJ, 435, L15

Marco, O., \& Alloin, D. 2000, A\&A, 353, 465

Marco, O., \& Brooks, K. J. 2003, A\&A, 398, 101

Micono, M., Bodo, G., Massaglia, S., et al. 2000, A\&A, 360, 795

Micono, M., Massaglia, S., Bodo, G., Rossi, P., \& Ferrari, A. 1998a, A\&A, 333, 989

Micono, M., Massaglia, S., Bodo, G., Rossi, P., \& Ferrari, A. 1998b, A\&A, 333, 1001
Mugnier, L. Z., Fusco, T., \& Conan, J. 2004, JOSAA, 21, 1841

Nenkova, M., Ivezić, Ž., \& Elitzur, M. 2002, ApJ, 570, L9

Ogle, P. M., Brookings, T., Canizares, C. R., Lee, J. C., \& Marshall, H. L. 2003, A\&A, 402, 849

Prieto, M. A., Meisenheimer, K., Marco, O., et al. 2004, ApJ, 614, 135

Rouan, D., Rigaut, F., Alloin, D., et al. 1998, A\&A, 339, 687

Rouan, D., Lacombe, F., Gendron, E., et al. 2004, A\&A, 417, L1

Rousset, G., Lacombe, F., Puget, P., et al. 2000, in Adaptive Optical Systems Technology, ed. P. L. Wizinowich, Proc. SPIE, 4007, 72

Saxton, C. J., Bicknell, G. V., \& Sutherland, R. S. 2002, ApJ, 579, 176

Schinnerer, E., Eckart, A., Tacconi, L. J., Genzel, R., \& Downes, D. 2000, ApJ, 533, 850

Simpson, J. P., Colgan, S. W. J., Erickson, E. F., et al. 2002, ApJ, 574, 95

Steffen, W., Gomez, J. L., Raga, A. C., \& Williams, R. J. R. 1997, ApJ, 491, L73

Thompson, R. I., Chary, R., Corbin, M. R., \& Epps, H. 2001, ApJ, 558, L97

Villar-Martín, M., De Young, D., Alonso-Herrero, A., Allen, M., \& Binette, L. 2001, MNRAS, 328, 848

Weigelt, G., Wittkowski, M., Balega, Y. Y., et al. 2004, A\&A, 425, 77

Wittkowski, M., Kervella, P., Arsenault, R., et al. 2004, A\&A, 418, L39 